\title{
Contributions to Gait Recognition Using Multiple-Views
}

\author{
David López-Fernández* \\ * Department of Computing and Numerical Analysis, University of Córdoba, Córdoba, Spain. \\ Thesis advisors: F.J. Madrid-Cuevas and A. Carmona-Poyato. \\ Date and location of PhD thesis defense: 13 May 2016, University of Córdoba.
}

Received 1st July 2016; accepted 1st July 2016

\begin{abstract}
This thesis focuses on identifying people by the way they walk. The problem of gait recognition has been addressed by using different approaches, both in the 2D and 3D domains, and using one or multiple views. However, the dependence on camera viewpoint (and therefore the dependence on the trajectory of motion) still remains an open problem. This dissertation addresses the problem of dependence on the trajectory through the use of 3D reconstructions of walking humans. The use of 3D models have several advantages that are worth mentioning. First, by the use of 3D reconstructions it is possible to exploit a greater amount of information in contrast to methods that extract descriptors from just 2D images. Second, the 3D reconstructions can be aligned along the way as if the subject had walked on a treadmill, thus providing a way to recognize people regardless the path. Three approaches are proposed in order to address the dependence on the trajectory: 1) using aligned 3D reconstructions of walking humans; 2) using unaligned 3D reconstructions of walking humans; 3) extracting a 3D description without using 3D reconstructions.

Three gait descriptors are also proposed. The first focuses on describing gait by means of morphological analysis of 3D aligned volumes [1]. The second makes use of the concept of entropy to describe the dynamics of human gait [2] (see Fig. 1). The third aims to capture the dynamics of gait in a rotation invariant way, which makes it interesting for recognize people walking on both straight and curves path, and regardless direction changes [3, 4] (see Fig. 2).

These approaches have been tested on the "AVA Multi-View Dataset (AVAMVG)" [5] and on the "Kyushu University 4D Gait Database (KY4D)". Both databases are specifically designed to address the problem of dependence on the viewpoint, and therefore the dependence on the trajectory. Experimental results show that for the approach based on aligned volumetric reconstructions, the entropy-based gait descriptor achieved the best results compared to other closely related methods of the state-of-art. However, the rotation invariant gait descriptor achieves a recognition rate that overcomes the compared state-of-art methods without requiring the alignment of the 3D gait reconstructions.
\end{abstract}

Correspondence to: <i52lofed@uco.es>

Recommended for acceptance by Dr. David Vázquez Bermúdez

DOI:http://dx.doi.org/10.5565/rev/elcvia.946

ELCVIA ISSN:1577-5097

Published by Computer Vision Center / Universitat Autònoma de Barcelona, Barcelona, Spain 


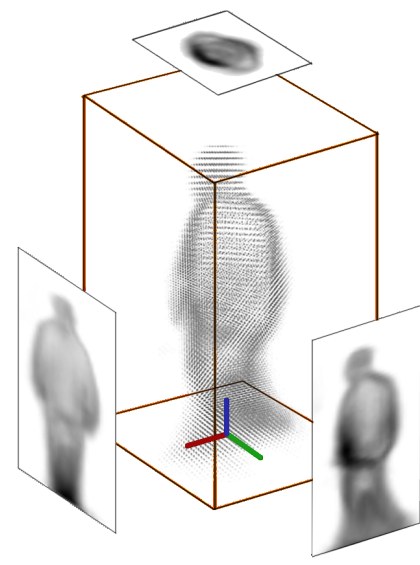

Figure 1: A 3D representation of the Gait Entropy Volume (GEnV) [2] computed over $L$ 3D-reconstructed and aligned volumes is shown in the center of the figure. Voxels are represented as points. Intensity on gray level represent the entropy value corresponding to that voxel. Marginal distributions of GEnV are also shown.
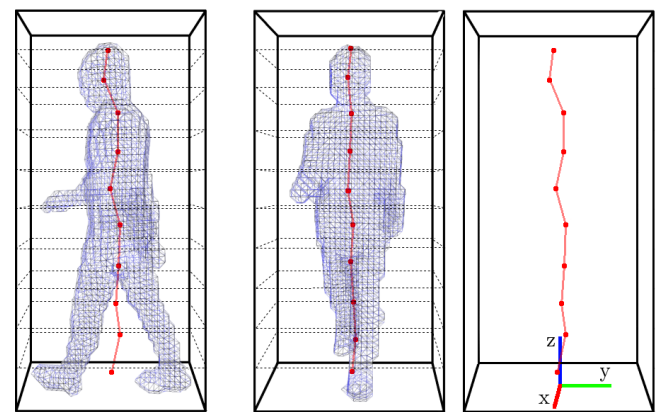

Figure 2: The reconstructed model is divided into 3D stacked areas of the same size called slices (regions within dotted lines). Centroids are computed on each slice (red points). The gait feature is composed by a set of inner angles between the line joining each pair of consecutive centroids (red line) and the Z-axis in $\mathbb{R}^{3}$ [3].

\section{References}

[1] D. López-Fernández, F. Madrid-Cuevas, A. Carmona-Poyato, M. Marín-Jiménez, R. Muñoz Salinas, and R. Medina-Carnicer, "Viewpoint-independent gait recognition through morphological descriptions of 3d human reconstructions," Image and Vision Computing, vol. 4849, pp. 1 - 13, 2016.

[2] D. López-Fernández, F. Madrid-Cuevas, A. Carmona-Poyato, R. Muñoz Salinas, and R. Medina-Carnicer, "Entropy volumes for viewpoint-independent gait recognition," Machine Vision and Applications, vol. 26, no. 7, pp. 1079-1094, 2015.

[3] D. López-Fernández, F. Madrid-Cuevas, A. Carmona-Poyato, R. Muñoz Salinas, and R. Medina-Carnicer, "A new approach for multi-view gait recognition on unconstrained paths," Journal of Visual Communication and Image Representation, vol. 38, pp. 396 - 406, 2016.

[4] D. López-Fernández, F. Madrid-Cuevas, A. Carmona-Poyato, R. Muñoz Salinas, and R. Medina-Carnicer, "Multi-view gait recognition on curved trajectories," in Proceedings of the 9th International Conference on Distributed Smart Cameras, ICDSC '15, (New York, NY, USA), pp. 116-121, ACM, 2015.

[5] D. López-Fernández, F. Madrid-Cuevas, A. Carmona-Poyato, M. Marín-Jiménez, and R. Muñoz Salinas, "The AVA Multi-View Dataset for Gait Recognition," in Activity Monitoring by Multiple Distributed Sensing, Lecture Notes in Computer Science, pp. 26-39, Springer International Publishing, 2014. 